\title{
An Unusual Case of Recurrent Migration of the Peritoneal Dialysis Catheter into the Inguinal Hernia Sac
}

\author{
Haruna Fukuzaki Junichiro Nakata Yuka Shirotani Yuki Shimizu \\ Masayuki Maiguma Nao Nohara Yusuke Suzuki \\ Department of Nephrology, Juntendo University Faculty of Medicine, Tokyo, Japan
}

\section{Keywords}

Peritoneal dialysis · Inguinal hernia $\cdot$ Polycystic kidney disease $\cdot$ Malposition of peritoneal dialysis catheter

\begin{abstract}
We herein report the first case of a patient with recurrent migration of the peritoneal dialysis $(P D)$ catheter into the inguinal hernia sac. A 58-year-old man suffered from end-stage renal disease due to polycystic kidney disease (PKD). A year before starting PD, a PD catheter was implanted with stepwise initiation of PD using the Moncrief-Popovich technique. He complained of drain failure and right inguinal swelling during the induction period and was diagnosed with right inguinal hernia. Further examination revealed that the PD catheter tip had migrated into the inguinal hernia sac. Although surgery was planned, the PD catheter tip spontaneously migrated back into the intra-peritoneal space. 14 months later, he noticed fill and drain failure again. Diagnosis was PD catheter dysfunction due to migration into the right inguinal hernia sac. PD was resumed without issues after repositioning of the PD catheter and repair of the inguinal hernia. Inguinal hernia is a frequent complication in PD patients, especially in those with PKD. Early diagnosis and treatment of hernia should be considered in PD patients.
\end{abstract}

\section{Introduction}

Peritoneal dialysis (PD) is a strategy for renal replacement in patients with end-stage renal disease (ESRD), achieving survival rates comparable to those of hemodialysis (HD) while preserving residual renal function. Of note, PD also is a cost-effective home-based 
therapeutic modality. As the elderly population increases, the demand for home-based therapy increases, and PD has been receiving more attention worldwide.

Inguinal hernia is a common noninfectious complication of PD [1]. In addition, patients with polycystic kidney disease (PKD) are believed to be at high risk for inguinal hernia development $[2,3]$. Inguinal hernia is troublesome because it sometimes leads to discontinuation of PD and change to HD. Some patients suffer life-threatening hernias, with intestinal incarceration or strangulation $[1,4]$. Prompt diagnosis and treatment of this disease are essential for continuing PD safely in the patients. We present an unusual case of PD catheter dysfunction due to recurrent migration of the PD catheter tip into the inguinal hernia sac, which was successfully managed.

\section{Case Presentation}

A 58-year-old male had ESRD due to PKD. His type 2 diabetes mellitus and an intraductal papillary mucinous neoplasm, a pancreatic cystic tumor, had been stable for years. He had no valve disease or cerebral aneurysm as complications of PKD. He chose PD for renal replacement therapy and underwent implantation of a straight PD catheter (Long Shoot Catheter 650 with reinforcement JL-1(A)S ${ }^{\circledR}$; Hayashidera Medinol, Kanazawa, Japan) with stepwise initiation of PD using the Moncrief-Popovich technique, which was developed to reduce catheter-related infection in PD patients [5]. The method includes the surgical implantation of the catheter, with its end buried in subcutaneous tissue until the initiation of PD.

PD was initiated due to extreme azotemia 1 year after the implantation of the PD catheter. The 4-h dialysate/plasma creatinine ratio was 0.86 by the peritoneal equilibration test, and the patient's small-molecule transport status was categorized as high at PD initiation. Although high transporters usually manage extracellular fluid volume better with short dwell times, continuous ambulatory PD with 3 daily exchanges (1,500 mL Reguneal HCa $1.5 \%{ }^{\circledR}$; Baxter, Tokyo, Japan) was chosen in the present patient due to lifestyle constraints. A month after the PD initiation, icodextrin was added due to a 3 -kg weight gain and worsening peripheral edema (Fig. 1).

Two months after the introduction of icodextrin, he complained of drain failure while in the dorsal position as well as a right inguinal swelling. The patient denied abdominal pain, nausea, vomiting, or constipation. Physical examination demonstrated a soft, nontender, reducible mass in the standing posture, which disappeared in the dorsal position. CT demonstrated a $30 \times 45$ $\mathrm{mm}$ cystic lesion in the right inguinal region and the PD catheter tip in that space (Fig. 2a). The laboratory data were mostly unremarkable except for blood urea nitrogen (56 mg/dL), creatinine $(12.53 \mathrm{mg} / \mathrm{dL})$, and $\beta 2$-microglobulin $(18.0 \mathrm{mg} / \mathrm{L})$. Diagnosis was a right inguinal hernia and PD catheter malposition. Although surgical hernia repair was scheduled, the PD catheter tip spontaneously migrated back into the pelvic cavity (Fig. 2b). After the observation of drain improvement, the patient was switched to nightly automated PD, with 3 exchanges during sleep $\left(1,500 \mathrm{~mL}\right.$ Dianeal $1.5 \%{ }^{\circledR}$; Baxter, Tokyo, Japan), to secure a dry day time due to inguinal hernia (Fig. 1). After the resolution of fill and drain failure, the patient refused to undergo hernioplasty due to a busy work schedule. Therefore, surgery to repair the inguinal hernia was canceled.

At 14 months later, he presented again with fill and drain failure. CT with the patient supine revealed the PD catheter tip in the right inguinal hernia sac (Fig. 2c, d) with no definitive bowel loops in the space. Diagnosis was PD catheter dysfunction due to recurrent migration of the PD catheter tip into the right inguinal hernia sac. Temporary HD was performed, and the PD catheter was repaired via the forefinger procedure [6]. He resumed PD after an abdominal radiograph confirmed the tip of the PD catheter to be repositioned in the pelvic floor. One month after PD catheter repair, an open mesh hernioplasty was performed. The hernia has not recurred during 20 months of follow-up.

\section{Karger'}




\section{and Dialysis}

\begin{tabular}{l|l}
\hline DOI: 10.1159/000515566 & $\begin{array}{l}\text { ( ) 2021 The Author(s). Published by S. Karger AG, Basel } \\
\text { www.karger.com/cnd }\end{array}$ \\
\hline
\end{tabular}

Fukuzaki et al.: Recurrent Migration of Peritoneal Dialysis Catheter into Inguinal Hernia Sac

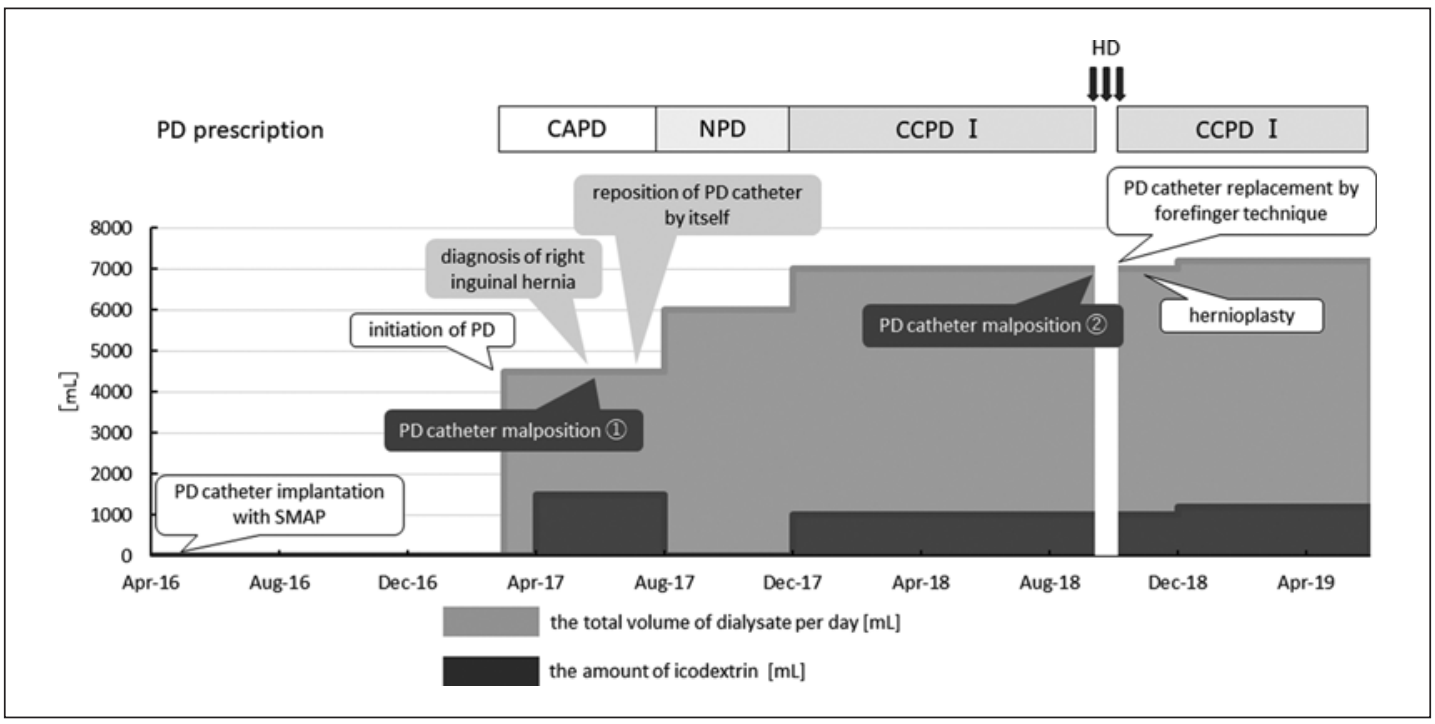

Fig. 1. The clinical course and change in total dialysate volume and icodextrin. HD, hemodialysis; CAPD, continuous ambulatory peritoneal dialysis; NPD, nightly peritoneal dialysis; CCPD, continuous cyclic peritoneal dialysis; SMAP, stepwise initiation of PD using the Moncrief-Popovich technique.
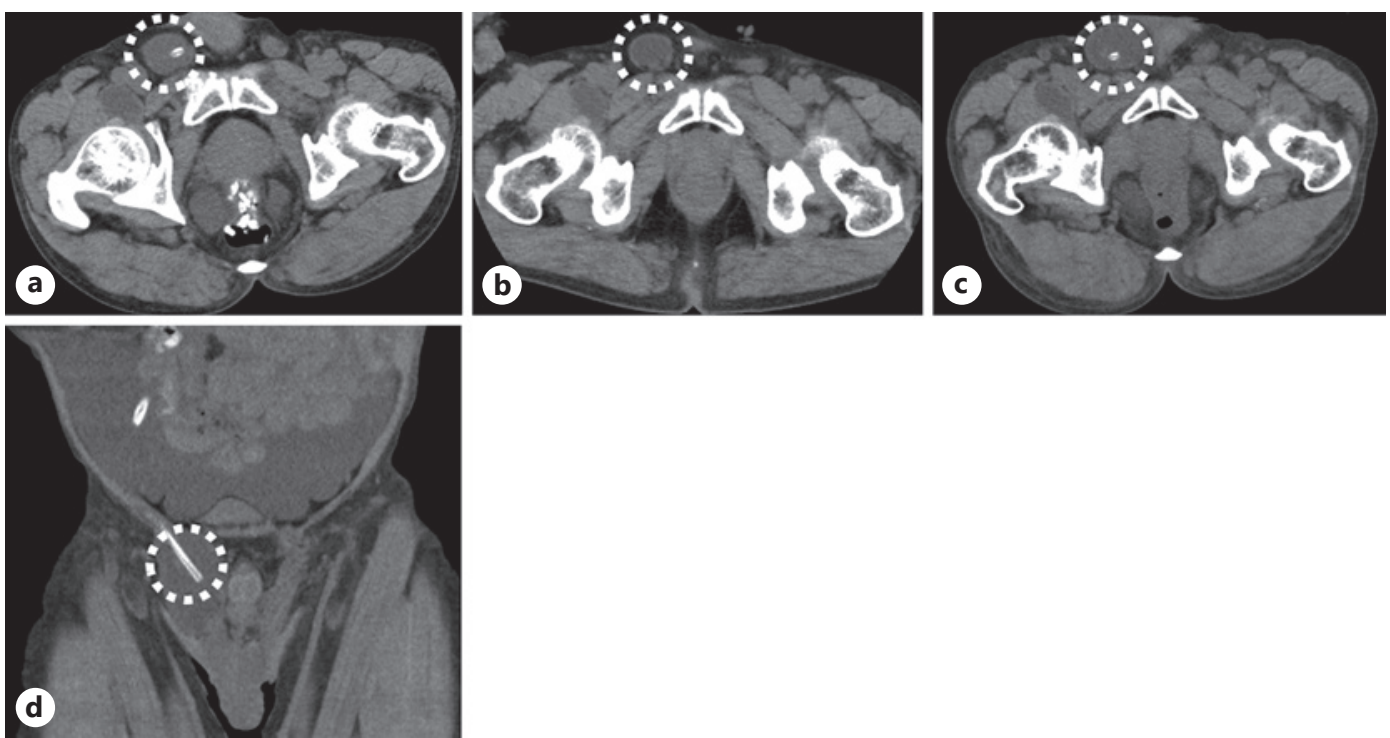

Fig. 2. a CT showing that the tip of the PD catheter is invading into the right inguinal hernia. $\mathbf{b}$ The PD catheter tip repositioned in the intraperitoneal cavity. $\mathbf{c}, \mathbf{d}$ CT showing recurrent PD catheter tip migration into the right inguinal hernia sac. PD, peritoneal dialysis.

\section{Discussion}

Inguinal hernia is a common noninfectious complication in PD patients. In particular, Rocco and Stone [1] reported inguinal hernias in $4-14 \%$ of patients after PD, and this incidence is higher than the $1.5 \%$ incidence of inguinal hernia in the general population. PKD and advanced age, high body mass index (BMI), and history of abdominal operation are independent risk factors for hernia development $[2,3]$. 
Hamanoue et al. [2] suggested that performance of PD can be affected by renomegaly and hepatomegaly in patients with PKD. The enlarged kidneys and liver decrease the effective intra-abdominal space and increase intra-abdominal pressure. Some reports showed that these conditions sometimes lead to inadequate dialysis and a high incidence of pleural effusion, dialysate leakage, and abdominal hernia [3]. Therefore, PKD often has been considered a relative contradiction to PD. On the other hand, Kumar et al. [7] found no difference in long-term outcome of PD therapy in patients with PKD compared to other nondiabetic patients. Recent reports also show that PD does not have a negative impact on overall survival and PD technique failure in patients with PKD and concluded that PD is a reasonable option for patients with PKD [8]. For these reasons, managing the complications related to increasing intraperitoneal pressure is important.

By injecting 1,000 mL dialysate, intra-abdominal pressure increases to 2.0, 2.7, and 2.8 $\mathrm{cm}_{2} \mathrm{O}$ in the supine, standing, and sitting positions, respectively [9]. There also is strong linear correlation between intra-peritoneal pressure and intraperitoneal volume [10]. Furthermore, Tokgöz et al. [11] reported that patients with small body size tended to have an increased risk for hernia development and that body height, body weight, body surface area, and total body water for patients could be used to estimate the risk of hernia. Our patient might have been at high risk for hernia development because of his PKD and high volume of PD dialysate during the daytime compared to his small body surface area.

There are some variations in the design of PD catheters. In regard to the shape of intraabdominal segments, straight catheters are supposed to be more prone to migration into the hernia sac than coiled catheters. However, Xie et al. [12] reported that coiled catheters were significantly associated with increased risk of catheter tip migration and resultant dysfunction. Our center usually uses the catheter with a straight intra-abdominal portion to reduce the risk of mechanical complications.

Inguinal hernia is a familiar condition worldwide, and it also is a common complication in PD patients. Hernia has been reported previously to lead to PD discontinuation and change to HD and could cause life-threatening complications, such as bowel incarceration or strangulation [1]. There are some similar reports in the past. Francis et al. [13] and Teoh et al. [14] presented the cases of PD catheter dysfunction by migration into a hernia sac, in which the patient underwent reposition of the PD catheter and hernia repair. Alsina et al. [15] reported that the PD catheter tip, which had got stuck into a hernia sac, migrated back into the pelvic cavity without specific maneuvers, and the patient was subsequently treated with open herniorrhaphy to prevent recurrence of PD catheter migration into the space. Our case is the first reported patient with "recurrent" migration of the PD catheter into the right inguinal hernia sac. At the initial PD catheter malposition, he presented with failure to drain only (ability to fill was retained), and the catheter fortunately spontaneously migrated back into the abdominal space. For this reason, the patient postponed treatment for his hernia. However, at the second presentation, he had fill and drain failure, requiring surgery to replace the PD catheter. If he had undergone hernia repair soon after the first migration, he might have avoided the invasive procedure. Prompt detection of the inguinal hernia and performing surgical treatment without hesitation is necessary to avoid complications and continue PD safely.

\section{Conclusion}

We presented an unusual case of recurrent migration of the PD catheter into the inguinal hernia sac. Surgical treatment for the inguinal hernia should be considered as soon as diagnosis is made in PD patients. For continued safe PD, the importance of early diagnosis and prompt treatment for the inguinal hernia should be emphasized.

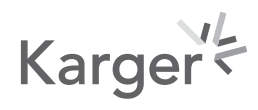




\section{Case Reports in Nephrology and Dialysis}

\begin{tabular}{l|l}
\hline Case Rep Nephrol Dial 2021;11:152-157 \\
\hline DOI: 10.1159/000515566 & $\begin{array}{l}\text { @ 2021 The Author(s). Published by S. Karger AG, Basel } \\
\text { www.karger.com/cnd }\end{array}$ \\
\hline
\end{tabular}

Fukuzaki et al.: Recurrent Migration of Peritoneal Dialysis Catheter into Inguinal Hernia Sac

\section{Statement of Ethics}

Written informed consent was obtained from the patient for publication of this case report and all accompanying images.

\section{Conflict of Interest Statement}

The authors have no conflicts of interest to declare.

\section{Funding Sources}

The authors received no specific funding for this work.

\section{Author Contributions}

H.F. conceived the article and drafted the manuscript. Y.S., Y.S., M.M., and N.N. followed up the patient as an outpatient. J.N. contributed to the operation for PD catheter repairment and revised the article critically for important intellectual content. Y.S. reviewed the manuscript and gave approval of the final version to be submitted.

\section{References}

1 Rocco MV, Stone WJ. Abdominal hernias in chronic peritoneal dialysis patients: a review. Perit Dial Int. 1985 Jul;5(3):171-4.

2 Hamanoue S, Hoshino J, Suwabe T, Marui Y, Ueno T, Kikuchi K, et al. Peritoneal dialysis is limited by kidney and liver volume in autosomal dominant polycystic kidney disease. Ther Apher Dial. 2015 Jun;19(3):207-11.

3 De V, Scalamogna A, Scanziani R, Castelnovo C, Dozio B, Rovere G. Polycystic kidney disease and late peritoneal leakage in CAPD: are they related? Perit Dial Int. 2002 Jan-Feb;22(1):82-4.

4 Horvath P, Königsrainer A, Mühlbacher T, Thiel K, Thiel C. Hernia repair and simultaneous continuous ambulatory peritoneal dialysis (CAPD) catheter implantation: feasibility and outcome. Hernia. 2020 Aug;24(4): 867-72.

5 Moncrief JW, Popovich RP, Broadrick LJ, He ZZ, Simmons EE, Tate RA. The Moncrief-Popovich catheter. A new peritoneal access technique for patients on peritoneal dialysis. ASAIO J. 1993 Jan-Mar;39(1):62-5.

6 Sakurada T, Kohatsu K, Yamada S, Sato H, Kojima S, Koitabashi K, et al. Minilaparotomy for malfunctioning peritoneal dialysis catheter by nephrologists: experiences at two centers. Ren Replace Ther. 2020 Mar;6(1): 14.

7 Kumar S, Fan SL, Raftery MJ, Yaqoob MM. Long term outcome of patients with autosomal dominant polycystic kidney diseases receiving peritoneal dialysis. Kidney Int. 2008 Oct;74(7):946-51.

8 Sigogne M, Kanagaratnam L, Dupont V, Couchoud C, Verger C, Maheut H, et al. Outcome of autosomal dominant polycystic kidney disease patients on peritoneal dialysis: a national retrospective study based on two French registries (the French Language Peritoneal Dialysis Registry and the French renal epidemiology and information network). Nephrol Dial Transplant. 2018 Nov;33(11):2020-6.

9 Twardowski ZJ, Prowant BF, Nolph KD, Martinez AJ, Lampton LM. High volume, low frequency continuous ambulatory peritoneal dialysis. Kidney Int. 1983 Jan;23(1):64-70.

10 Dejardin A, Robert A, Goffin E. Intraperitoneal pressure in PD patients: relationship to intraperitoneal volume, body size and PD-related complications. Nephrol Dial Transplant. 2007 May;22(5):1437-44.

11 Tokgöz B, Dogukan A, Güven M, Unlühizarci K, Oymak O, Utas C. Relationship between different body size indicators and hernia development in CAPD patients. Clin Nephrol. 2003 Sep;60(3):183-6.

12 Xie J, Kiryluk K, Ren H, Zhu P, Huang X, Shen P, et al. Coiled versus straight peritoneal dialysis catheters: a randomized controlled trial and meta-analysis. Am J Kidney Dis. 2011 Dec;58(6):946-55.

13 Francis RS, D'Souza P, D'Souza RJ, Beaman M, Hamad S. An unusual cause of peritoneal dialysate drainage failure: inadvertent placement of tenckhoff peritoneal dialysis catheter into an occult inguinal hernia. Perit Dial Int. 2004 Jul-Aug;24(4):405-6. 
Case Reports

in Nephrology and Dialysis
Case Rep Nephrol Dial 2021;11:152-157

DOI: $10.1159 / 000515566$

(C) 2021 The Author(s). Published by S. Karger AG, Base www.karger.com/cnd

Fukuzaki et al.: Recurrent Migration of Peritoneal Dialysis Catheter into Inguinal Hernia Sac

14 Teoh CW, Haydar R, Gillick J, Waldron M, Dolan NM, Awan A, et al. Migration of tenckhoff catheter into an occult inguinal hernia. Perit Dial Int. 2015 Jan-Feb;35(1):113-4.

15 Alsina E, Julián Á, Millán I, Ramón JF, Sirvent AE, Alcaide MJ. “Self-locating” peritoneal catheter displaced into an inguinal hernia. Perit Dial Int. 2015 Sep-Oct;35(5):602. 